\title{
Adesão à terapia imunossupressora de transplantados renais de um hospital universitário
}

\begin{abstract}
RESUMO
O estudo objetivou descrever a adesão ao uso de imunossupressores e fatores influenciadores de transplantados renais. Trata-se de um estudo transversal descritivo-exploratório quantitativo realizado em um hospital universitário do Estado do Rio de Janeiro que contou com 47 transplantados entre 2013 e 2017. Utilizou-se um formulário para coleta de dados sociodemograficos e para avaliação da adesão à terapia imunossupressora aplicou-se o instrumento Basel Assessment of Adherence to Immunosuppressive Medications Scale BAASIS. Para coleta e análise dos dados foi criado um questionário no software Epilnfo®7.2. Conclusão: 0 trabalho evidenciou que a não adesão à terapia imunossupressora é um problema importante devido ao seu potencial de perda de enxerto renal e foi possível traçar os pontos de dificuldade entre os pacientes não aderentes e, através da escala BAASIS identificar os pontos- chave de limitação para então intervir estrategicamente, visando aumentar sobrevida do enxerto, melhorar a qualidade de vida e prestar assistência direcionada às mudanças necessárias.
\end{abstract}

DESCRITORES: Transplante de Rim; Adesão à Medicação; Imunossupressão.

\section{ABSTRACT}

The study aimed to describe the adherence to the use of immunosuppressants and factors that influence kidney transplantation. This is a descriptive and exploratory quantitative cross-sectional study conducted in a university hospital in the state of Rio de Janeiro, which included 47 transplant recipients between 2013 and 2017. A form was used to collect sociodemographic data and to assess adherence to immunosuppressive therapy. the Basel Assessment of Adherence to Immunosuppressive Medications Scale BAASIS instrument was applied. For data collection and analysis, a questionnaire was created using Epilnfo ${ }^{\circledR 7.2}$ software. Conclusion: The study showed that non-adherence to immunosuppressive therapy is an important problem due to its potential for renal graft loss and it was possible to trace the points of difficulty among non-adherent patients and, through the BAASIS scale, identify the key points of limitation to intervene strategically, aiming to increase graft survival, improve quality of life and provide targeted assistance for necessary changes.

KEYWORDS: Kidney Transplantation; Medication Adherence; Immunosuppression.

\section{RESUMEN}

El estudio tuvo como objetivo describir la adherencia al uso de inmunosupresores y los factores que influyen en el trasplante de riñón. Este es un estudio cuantitativo transversal descriptivo y exploratorio realizado en un hospital universitario en el estado de Río de Janeiro, que tuvo 47 receptores de trasplante entre 2013 y 2017. Se utilizó un formulario para recopilar datos sociodemográficos y evaluar la adherencia a la terapia inmunosupresora. Se aplicó el instrumento BAASIS de la Escala de Evaluación de Adhesión a Medicamentos Inmunosupresores de Basilea. Para la recopilación y análisis de datos, se creó un cuestionario utilizando el software Epilnfo®7.2. Conclusión: El estudio mostró que la no adherencia a la terapia inmunosupresora es un problema importante debido a su potencial de pérdida de injerto renal y fue posible rastrear los puntos de dificultad entre pacientes no adherentes y, a través de la escala BAASIS, identificar los puntos clave de limitación para intervenir estratégicamente, con el objetivo de aumentar la supervivencia del injerto, mejorar la calidad de vida y proporcionar asistencia específica para los cambios necesarios.PALABRAS CLAVE: Transplante de riñón; Cooperación del paciente; Cumplimiento de la medicación; Enfermería; Inmunosupresores.

PALABRAS CLAVE: Transplante de Riñón; Cumplimiento de la Medicación; Inmunosupresión.

RECEBIDO EM: 06/11/2019 APROVADO EM: 07/11/2019

\section{Anna Luiza Pereira Magalhães}

Especialista em Nefrologia pelo Programa de Residência de Enfermagem UERJ, Enfermeira. Universidade do Estado do Rio de Janeiro, Rio de Janeiro-RJ-Brasil.

\section{Tatiane da Silva Campos}

Doutoranda em Bioética, Ética Aplicada e Saúde Coletiva, Professora Assistente da Universidade do Estado do Rio de Janeiro, Rio de Janeiro-RJ-Brasil. 


\section{Viviane Ganem Kipper de Lima}

Mestre em Enfermagem, Professora Assistente da Universidade do Estado do Rio de Janeiro, Rio de Janeiro-RJ-Brasil.

\section{Silvia Maria de Sá Basílio Lins}

Doutora em enfermagem, Professora Assistente da Universidade do Estado do Rio de Janeiro, Rio de Janeiro-RJ-Brasil.

\section{Edison Souza}

Doutor em Medicina, Professor Adjunto, Universidade do Estado do Rio de Janeiro, Rio de Janeiro-RJ-Brasil.

\section{INTRODUÇÃO}

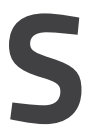
egundo a diretriz da Kidney Disease Outcome Quality Initiative (KDOQI), a pessoa com Doença Renal Crônica (DRC) é aquela que, "independente da causa, apresente Taxa de Filtração Glomerular $(\mathrm{TFG})<60 \mathrm{~mL} / \mathrm{min} / 1,73 \mathrm{~m} 2$ ou $>60 \mathrm{~mL} / \mathrm{min} / 1,73 \mathrm{~m} 2$ associada a pelo menos um marcador de dano renal parenquimatoso por 3 meses"(1). Uma vez diagnosticada a DRC em seu último estágio e havendo a necessidade de Terapia Renal Substitutiva (TRS), a pessoa iniciará uma das três modalidades existentes, sendo elas: diálise peritoneal, hemodiálise e transplante renal ${ }^{(1)}$.

$\mathrm{O}$ transplante renal é indicado como tratamento para pacientes com TFG menor que $15 \mathrm{ml} / \mathrm{min}$, estando ou não em tratamento dialítico ${ }^{(2)}$. Do ponto de vista clínico e social o transplante é a opção que oferece maior sobrevida, melhor qualidade de $\operatorname{vida}^{(3)}$ e, por vezes, é o único recurso para manutenção da vida do indivíduo ${ }^{(4)}$. O Censo da Sociedade Brasileira de Nefrologia de 2018 estima que 29.545 pacientes estejam em fila de espera para transplante renal $^{(5)}$ e 5.923 transplantes renais foram realizados, sendo 1.018 de doador vivo e 4.905 de doador falecido ${ }^{(6)}$.

Como toda terapêutica de doença crônica, o transplante necessita de boa adesão ao tratamento e uso dos imunossupressores, para que se obtenha o sucesso da manutenção da saúde e do enxerto a longo prazo(7). Segundo a Organização Mundial de Saúde (OMS), a adesão ao tratamento ${ }^{(8)}$ traz em seu conceito a capacidade do indi- víduo em agir conforme as orientações do profissional de saúde quanto à tomada dos medicamentos, estilo de vida e nutrição adequados.

Quanto à terapia imunossupressora, há dois componentes básicos envolvidos que caracterizam a não-adesão: a descontinuação do regime prescrito e a qualidade desse regime ${ }^{(9)}$. Vale ressaltar que a adesão é um comportamento mutável, portanto é flexível com tempo e sofre influência multifatorial, sendo necessária a abordagem individual a cada usuário como forma de compreender o que pode ser modificado e melhorado ${ }^{(10)}$.

Esse trabalho foi inspirado no Adere Brasil, um estudo ${ }^{(11)}$ multicêntrico, iniciado em 2014, que busca avaliar a não-adesão ao transplante renal e explorar os fatores influenciadores, bem como trocar informações entre os centros de atendimento ao paciente transplantado renal. Como o centro em questão não está incluído no estudo, realizou-se a pesquisa para aumentar o conhecimento sobre os centros transplantadores.

Diante do exposto surgiu a inquietação: Quais são os fatores que influenciam na adesão à terapia imunossupressora dos pacientes atendidos no ambulatório de pós-transplante renal? Este estudo tem como objetivo descrever a adesão ao uso de imunossupressores e fatores influenciadores de transplantados renais.

\section{METODOLOGIA}

Trata-se de um estudo transversal descritivo-exploratório, com abordagem quantitativa, realizado durante as consultas pré-agendadas, no ambula- tório de pós-transplante renal de um hospital universitário do Estado do Rio de Janeiro. O estudo incluiu todos os 47 transplantados renais no período de 2013 à 2017, de ambos os sexos; maiores de dezoito anos; atendidos regularmente no ambulatório de pós-transplante renal e que tivessem consulta agendada durante o período de coleta de dados, que ocorreu entre os meses de junho a novembro de 2018. Os critérios de exclusão foram: impossibilitados de responder aos questionários (incapacidades cognitivas ou auditivas pré-diagnosticadas pelo médico assistente).

A participação foi voluntária, seguindo os preceitos da Resolução n. ${ }^{\circ}$ 466, de 12 de dezembro de 2012 do Conselho Nacional de Saúde (CNS), mediante assinatura do Termo de Consentimento livre e Esclarecido (TCLE) após aprovação do Comitê de Ética em Pesquisa pelo Parecer n. ${ }^{\circ}$ 2.611.942.

$\mathrm{O}$ instrumento para coleta de dados sociodemográficos foi composto por variáveis que abordam os dados de sexo, idade, nível de escolaridade, religião, faixa salarial, atividades laborais e hábitos de vida. Aborda ainda as informações relacionadas ao tratamento (medicação e exames) e dificuldades pessoais.

Para a produção de dados sobre a adesão à terapia imunossupressora foi aplicada a escala The Basel Assessment of Adherence With Imunossupressive Medication Scale - BAASIS ${ }^{(12)}$, em sua versão traduzida e validada para a população brasileira. Os dados foram analisados por meio de estatística descritiva simples, a partir do banco de dados criado no software EpiInfo ${ }^{\circ} 7.2$. 
Tabela 1. Características sociodemográficas dos participantes. Rio de Janeiro, RJ, Brasil, 2018

\section{VARIÁVEIS}

ADERENTES

NÃO-ADERENTES

Idade

18-27 anos

28-37

38-47

48-57

58-67

68 ou +

\section{Escolaridade}

Analfabeto

Fundamental incompleto

Fundamental completo

Médio incompleto

Médio completo

Superior incompleto

Superior completo

\section{Situação empregatícia}

Aposentado

Pensionista

Do lar

Trabalho informal

Trabalho formal

Estudante

\section{Renda Mensal}

Até $1 \mathrm{SM}$

Entre 2 e 5 SM

Mais de 5 SM

TOTAL

$2(6 \%)$
$3(9 \%)$
$5(15.2 \%)$
$13(39.4 \%)$
$10(30.4 \%)$

0

$4(28.6 \%)$

$3(21.5 \%)$

$3(21.5 \%)$

$1(7.1 \%)$

$2(14.2 \%)$

$1(7.1 \%)$

$\begin{array}{cc}1(3 \%) & 0 \\ 2(6 \%) & 3(21.5 \%) \\ 6(18.2 \%) & 2(14.2 \%) \\ 5(15.2 \%) & 2(14.2 \%) \\ 14(42.5 \%) & 5(35.9 \%) \\ 1(3 \%) & 2(14.2 \%)\end{array}$

$4(12.1 \%)$

0

$\begin{array}{cc}7(21.2 \%) & 4(28.6 \%) \\ 2(6 \%) & 0 \\ 5(15.1 \%) & 3(21.4 \%) \\ 3(9 \%) & 1(7.1 \%) \\ 16(48.5 \%) & 4(28.6 \%) \\ 0 & 2(14.2 \%)\end{array}$

$8(24.3 \%)$

$6(42.9 \%)$

$22(66.7 \%)$

$6(42.9 \%)$

$3(9 \%)$

33

\section{RESULTADOS}

Dos 132 transplantados renais entre 2013 a 2017: 33 pacientes foram a óbito, 09 perderam o enxerto, 17 negaram participação, 15 faltaram à consulta, 07 foram eliminados por critério de exclusão; 31 pacientes não tiveram consulta agendada durante o período de coleta de dados; o que resultou, ao final, 47 usuários entrevistados. Destes, $14(29,8 \%)$ foram considerados não aderentes.

Amostra total foi bem distribuída entre homens $(59,6 \%)$ e mulheres $(40,4 \%)$, assim como entre os não-aderentes onde (08 homens e $06 \mathrm{mu}-$ lheres). Em relação à idade, a maior parcela dos participantes (29,7\%) se manteve na taxa dos 48-67 anos. Quanto aos não-aderentes a mediana foi de 39 anos, incluindo o membro mais novo (22 anos) e o membro mais velho (71 anos).

Quanto ao tratamento, $57,4 \%$ utilizam 5 ou mais medicamentos por dia, $59,6 \%$ não utilizam formas de lembrete para as tomadas de medicamentos e, $65,9 \%$ não tem quem auxilie a lembrar os horários. Todos os pacientes relataram comparecer com frequência à consulta, $97,9 \%$ relatou não ter faltado nos últimos 6 meses; 71,8\% realiza os exames solicitados pelo Sistema Único de Saúde (SUS). A retirada das medicações imunossupressoras é realizada na farmácia estadual por $91,5 \%$ dos usuários e $57,4 \%$ considera o local longe de sua residência. Para 70,2\% dos usuários esse local é fora de sua cidade de residência.

Os dados descritos na Tabela 2 mostram fatores de risco para complicações, como a inatividade física, tabagismo e etilismo. A necessidade de alerta quanto ao consumo de bebida alcoólica entre os pacientes transplantados fica evidenciada, pois $21,3 \%$ se declara como etilista e $30 \%$ ex-etilista. Diante disso, é preciso atentar uma vez que o consumo exagerado de álcool pode levar à descontinuidade do tratamento $\mathrm{e}$ agravo do quadro clínico. 


\section{artigo}

Magalhães, A.L.P.; Campos, T.S.; Lima, V.G.K.; Lins, S.M.S.B.; Souza, E.;

Adesão à terapia imunossupressora de transplantados renais de um hospital universitário
Ex-etilista
$11(33.3 \%)$
$3(21.4 \%)$
Nunca
$16(48.5 \%)$
$9(64.4 \%)$
33
14

TOTAL

Tabela 3. Dados relacionados a TRS e comorbidades. Rio de Janeiro, RJ, Brasil, 2018

\section{VARIÁVEIS}

ADERENTES

NÃO-ADERENTES

Tempo de diálise que realizou

0

Até 12 meses

$4(12.1 \%)$

$2(14.3 \%)$

$2(6 \%)$

0

Até 60 meses

$14(42.5 \%)$

$9(64.4 \%)$

Até 120 meses

$10(30.4 \%)$

$1(7.1 \%)$

Até 180 meses

$2(6 \%)$

$1(7.1 \%)$

$>180$ meses

$1(3 \%)$

$1(7.1 \%)$

\section{Tipo de doador}

Cadáver

$23(69.7 \%)$

$8(57.2 \%)$

Vivo

$10(30.3 \%)$

$6(42.8 \%)$

Transplante pré-emptivo

Não

$29(87.9 \%)$

$12(85.7 \%)$

Sim

$4(12,1 \%)$

$2(14,3 \%)$

Foi internado após o transplante?

Sim

$17(51.5 \%)$

$9(64.3 \%)$

Não

$16(48,5 \%)$

$5(35,7 \%)$

Possui comorbidades? *

Não

$5(15.1 \%)$

$7(50 \%)$

Hipertensão

$27(81.8 \%)$

$6(42.8 \%)$

Diabetes

$8(24.2 \%)$

$4(28.6 \%)$

TOTAL
Sobre os dados do ambulatório e relacionamento com a equipe, todos os pacientes têm consultas agendadas frequentemente, sendo a frequência trimestral a mais comum $(72,3 \%)$ e, $91,5 \%$ declara não ter dificuldade para agendar consulta.

Quanto aos dados da escala BAASIS, destaca-se que dentre os 14 não aderentes, $35.7 \%$ respondeu a mais de 1 questão como positivo, reforçando a dificuldade de adesão em mais de um fator; e $85,7 \%$ relatam se lembrar de ter tomado seus remédios imunossupressores com mais de 2 horas de diferença em relação ao horário prescrito, nas últimas 4 semanas, conforme observa-se no Quadro 1.

\section{DISCUSSÃO}

Não há um consenso sobre a diferença entre homens e mulheres em relação à adesão ao tratamento medicamentoso $^{(13)}$. A literatura indica que pacientes com mais idade têm melhor adesão às terapias ${ }^{(14)}$. Apesar da mediana do grupo dos não-aderentes ser baixa e os 02 membros com menor idade entre os entrevistamos estarem nesse grupo, observamos que apenas $14,8 \%$ com 48 anos ou mais de idade estavam no grupo de não aderentes.

A literatura relaciona a baixa escolaridade como fator de risco para não-adesão ${ }^{(15)}$. Entre os não aderentes, a escolaridade está bem distribuída, e 50,1\% declaram ensino médio (completo e

Nota: Destaca-se que o paciente poderia responder que possuía mais de uma comorbidade dentre as listadas.

Quadro 1. Análise da escala de adesão. Rio de Janeiro, RJ, Brasil, 2018

DISTRIBUIÇÃO DA PONTUAÇÃO POR ITEM DA ESCALA BAASIS/NÚMERO DE PACIENTES RESPONDENTES

Item 1: Você se lembra de não ter tomado seus remédios imunossupressores alguma vez nas últimas 4 semanas?

Item 2: Você deixou de tomar doses consecutivas de sua medicação imunossupressora nas últimas 4 semanas?

Item 3: Você se lembra de ter tomado seus remédios imunossupressores com mais de 2 horas de diferença em relação ao horário prescrito, nas últimas 4 semanas?

Item 4: Você tomou uma dose menor do que a dose prescrita pelo seu médico, nas últimas 4 semanas?

6

1

12

2 
incompleto), não sendo possível estabelecer qualquer afirmação. Dos participantes com renda maior que 5 salários, $40 \%$ estão caracterizados como não aderentes. No entanto, apenas $24,3 \%$ dos aderentes possuem renda até 1 salário contra $42,8 \%$ dos não aderentes, dados que vão ao encontro com o descrito na literatura que correlaciona baixa adesão à baixa renda ${ }^{(16)}$.

Ao avaliar o parâmetro religioso, não foi especificado qual a religião, uma vez que a literatura descreve como fator influenciador no tratamento a espiritualidade ${ }^{(17)}$, aspecto capaz de estar presente independente de qual religião. Entre os aderentes, $93.9 \%$ afirmaram ter uma religião, entre os não aderentes foram $92.8 \%$, o que dificulta estabelecer relação entre os fatores.

A polifarmácia - o uso de 5 ou mais medicamentos ao dia ${ }^{(18)}$, é uma realidade pós-transplante renal devido aos esquemas profiláticos que, em conjunto com os tratamentos das doenças de base como hipertensão e diabetes, totalizam um montante de comprimidos que contribuem para ocorrência de reações adversas, dificuldade na tomada correta e, consequente, baixa adesão ${ }^{(19)}$, fato visto no perfil de pacientes do estudo.

Opções podem ser adotadas para não haver esquecimento das tomadas de medicamentos como lembretes no celular ou ajuda de algum parente, mecanismo crescente para auxiliar a adesão ${ }^{(20)}$. Ainda assim, 40\% dos que utilizam lembrete e $15 \%$ dos que possuem ajuda de alguém são não aderentes. Dessa forma, é possível perceber que, ainda com o uso de tecnologias e pessoas auxiliares, os recursos só serão úteis se o paciente realiza a tomada do medicamento ao ser alertado.

Entre os não aderentes, $42,8 \%$ afirmaram que o local de retirada é longe de casa, porém apenas $21,4 \%$ retiravam em outra cidade. Vale ressaltar que $75 \%$ dos pacientes que retiram o medicamento fora de sua cidade fo- ram considerados aderentes. Assim, é possível entender que, apesar do local de retirada ser um potencial problema para a adesão (pela dificuldade de acesso à medicação), a tomada de medicamentos ainda está mais relacionada ao comprometimento com as tomadas das doses nos horários corretos.

Diversos aspectos advindos do tempo prolongado de terapia podem afetar a adesão, por exemplo, alguma estabilidade da condição de saúde que estimula a negligência ao uso da medicaçãa ${ }^{(21)}$. Considerando que a coleta de dados abrangeu os pacientes dos últimos cinco anos, o período é muito similar entre eles, dificultando relacionar aqueles com tempo mais prolongado, visto que não são parte da amostra.

Entre os não aderentes, a amostra se dividiu entre os anos de 2013 (28,5\%), $2014(28,5 \%)$ e 2017 (42,8\%). É possível considerar que $57 \%$ dessa população foram transplantados há mais de 4 anos, porém relacionar o dado ao achado na literatura precisaria de uma amostra maior para sustentar este pressuposto.

Observamos como não aderentes, $37,5 \%$ dos receptores de doador vivo e $25,8 \%$ dos receptores de doador cadáver. Os dados são congruentes com o descrito na literatura, onde Jindal ${ }^{(22)}$ afirma que receptores de doador vivo são menos aderentes. Brahm ${ }^{(9)}$ atribui tal evento ao fato do transplante com doador vivo apresentar menos complicações e risco de rejeição, levando o receptor à menor preocupação com o tratamento.

Em relação à atividade física, o tratamento dialítico pode colaborar para a perda de massa magra e diminuição da disposição para exercício físico ${ }^{(23)}$, logo, é esperada a melhora desses fatores após o transplante renal. A prática de atividade física é bem distribuída no grupo de participantes e a maioria $(61,7 \%)$ realiza alguma atividade, sendo a mais prevalente a caminhada.

Outro fator importante para adesão é o bom relacionamento com a equi- pe de saúde. A criação de vínculo de modo a fornecer espaço seguro de escuta permite que o paciente sinta-se confortável e compreendido dentro das suas necessidades, evidenciando o relacionamento interpessoal como fator capaz de afetar a qualidade do tratamento para melhor ${ }^{(24)}$. Os resultados mostraram que houve frequência $\geq 89 \%$ na classificação "boa" quando ao relacionamento com os membros da equipe: médicos, enfermeiros e nutricionistas em todas as entrevistas.

Entre os não aderentes, 35,7\% dialisaram por um período menor que 2 anos, sendo este um dado descrito na literatura de que, quanto menor o tempo de diálise anterior ao transplante, maior o risco de não-adesão $0^{(9)}$.

Segunda a epidemiologia da DRC, cerca de $63 \%$ dos pacientes possuem hipertensão e diabetes como comorbidades ${ }^{(25)}$, dado que se evidencia também nesse estudo e pode colaborar para a menor adesão uma vez que contribui para a polifarmácia e maior risco de piora da função renal, merecendo atenção da equipe de saúde para o controle das mesmas.

Através da escala BAASIS, observamos que os pacientes relatam maior dificuldade para lembrar de tomar os medicamentos e usar no horário prescrito. Vale lembrar que os pacientes pontuaram em mais de um item da escala. Cabe ao enfermeiro reforçar as alternativas que auxiliam o paciente a tomar a medicação no horário certo, sempre enfatizando que a adesão à terapia imunossupressora é parte fundamental para que não haja rejeição do enxerto ${ }^{(26)}$.

Alguns fatores podem afetar a tomada de medicações de maneira consciente, como os efeitos colaterais ${ }^{(27)}$, o que leva à reflexão de que os pacientes transplantados devem ser avaliados com frequência, pois, apesar do consenso de que o transplante traz melhor qualidade de vida quando permite o retorno às atividades habituais, o conceito de bem-estar é individual e o re- 
torno à diálise pode representar alívio dos sintomas que levam à não-adesão medicamentosa ${ }^{(28)}$.

\section{CONCLUSÃO}

A não adesão à terapia imunossupressora é um problema importante devido ao seu potencial de perda de enxerto renal. Ao coletar os dados, foi possível traçar os pontos de dificuldade entre os pacientes não aderentes e, através da escala BAASIS aplicada, identificar os pontos-chave de limitação para então intervir estrategicamente.

Como pontos fortes, identificamos que o trabalho trará contri- buições para o serviço com fatores de alerta para a atuação da equipe como, por exemplo, o alto número de etilistas, considerando a amostra e a clientela que se trata; e destacamos o rigor na busca dos pacientes durante os dias de agendamento de consultas ao ambulatório. Nenhum paciente agendado deixou de participar por falta de contato.

As limitações do estudo são o número de perdas (por óbito, perda de enxerto, negar participação, falta à consulta, eliminação por critério de exclusão e sem agendamento durante o período de coleta de dados), que culminou em um pequeno quantitativo de não aderentes e não haver todos os dados do Adere
Brasil disponíveis para correlacionar os achados, uma vez que o estudo foi inspirado nesse. Destacamos que, os dados da pesquisa multicêntrica já publicados concordam com aqueles encontrados nesse estudo como renda mais alta entre os não-aderentes ${ }^{(29)} \mathrm{e}$ o consumo de bebida alcoólica ${ }^{(30)}$.

Ressaltamos que esse estudo foi de suma importância para entendermos melhor os fatores que influenciam na adesão dos transplantados renais e, através deles, podermos pensar em formas de melhorar o cuidado ofertado, visando aumentar sobrevida do enxerto, melhorar a qualidade de vida e prestar assistência direcionada às mudanças necessárias. *

\section{REFERÊNCIAS}

1. National Kidney Foundation. KDOQI clinical practice guideline for hemodialysis adequacy: 2015 update. Am J Kidney Dis [Internet]. 2015 [acesso em 19 nov 2017]; 66(5):884-930. Disponivel em: http://www.ajkd.org/article/S0272-6386(15)01019$7 /$ pdf.

2. Ministério da Saúde, Secretaria de Atenção à Saúde, Departamento de Atenção Especializada e Temática (BR). Diretrizes Clínicas para o Cuidado ao paciente com Doença Renal Crônica - DRC no Sistema Único de Saúde/ Ministério da Saúde [Internet]. Brasília: Ministério da Saúde, 2014 [acesso em 29 ago 2017]. p.: 37 p.: il. Disponivel em: http://portalarquivos.saude. gov.br/images/pdf/2014/maio/09/Nefrologia-Diretrizes-cl--nicas-para-o-cuidado-ao-paciente-com-doen--a-renal-cr--nica--DRC-.pdf.

3. Gouveia DS, et al. Analysis of economic impact between the modality of renal replacement therapy. Jornal Brasileiro de Nefrologia AHEAD [Internet]. 2017 [acesso em 13 nov 2017]. Disponivel em: http://www.scielo.br/scielo.php?pi$d=S 0101-28002017001301102 \&$ script=sci_arttext\&tlng=pt. 4. Mendonça AEO, et al. Mudanças na qualidade de vida após transplante renal e fatores relacionados. Acta Paulista de Enfermagem [Internet]. 2014 [acesso em 19 nov 2017]; 27(3). Disponivel em: http://www.redalyc.org/html/3070/307031542016/. 5. Sociedade Brasileira de Nefrologia. CENSO DE DIÁLISE, SBN 2018. São Paulo (SP), 2019

6. ABTO. Registro Brasileiro de Transplantes Estatística de Transplantes [Internet]. 2018 [acesso em 13 set 2019]. Disponivel em: http://www.abto.org.br/abtov03/Upload/file/ RBT/2018/Lv_RBT-2018.pdf.

7. Fuzinatto $\bar{C}$, Delagnolli R, Marin SM, Maissiat GS. Adhesión al tratamiento inmunosupresor en pacientes después del trasplante renal: estudio descriptivo-exploratorio. Online braz. j. nurs. [Internet]. 2013 [acesso em 24 nov 2017]. Dis- ponível em: http://saudepublica.bvs.br/pesquisa/resource/ pt/bde-25690.

8. Gusmão JL, Mion-Junior D. Adesão ao tratamento - conceitos Adherence to the treatment - concepts. Rev Bras Hipertens [Internet]. 2006 [acesso em 09 out 2017]; 13(1): 23-25. Apud. Haynes RB. Determinants of compliance:The disease and the mechanics of treatment. Baltimore MD, Johns Hopkins University Press, 1979. Disponível em: https://www.researchgate.net/profile/Decio_Mion2/publication/237662499_Adesao_ao_tratamento___conceitos_Adherence_to_the_treatment_-_concepts/links/00b4953b43021ebbfc000000/ Adesao-ao-tratamento-conceitos-Adherence-to-the-treatment-concepts.pdf.

9. Brahm MMT, Gonçalves LFS. Adesão aos imunossupressores em pacientes transplantados renais. [Dissertação] Mestrado em Medicina: Ciências Médicas - Universidade Federal do Rio Grande do Sul [Internet]. 2012 [acesso em 25 ago 2017]. Disponivel em: http://www.lume.ufrgs.br/handle/10183/39673.

10. Rebafka A. Medication Adherence After Renal Transplantation-a Review of the Literature. Journal of renal care [Internet]. 2016 [acesso em 24 nov 2017]. Disponível em: http:// onlinelibrary.wiley.com/doi/10.1111/jorc.12181/full.

11. Sanders-Pinheiro HS, et al. Prevalence and correlates of non-adherence to immunosuppressants and to health behaviors in patients after kidney transplantation in Brazil - the ADHERE BRAZIL multicentre study: a cross-sectional study protocol. BMC Nephrology [Internet]. 2018 [acesso em 18 out 2018]; 19:41. Disponivel em: https://bmcnephrol.biomedcentral.com/articles/10.1186/s12882-018-0840-6.

12. Marsicano EO. Não aderência no pós-transplante renal: validação do instrumento the basel assessment of adherence with imunossupressive medication scale e condições associadas. 


\section{REFERÊNCIAS}

[Dissertação] Mestrado em Saúde Brasileira - Universidade Federal de Juiz de Fora, Juiz de Fora [Internet]. 2012 [acesso em 09 out 2017]. Disponivel em: https://repositorio.ufjf.br/jspui/ handle/ufjf/1740.

13. Boucquemont J, et al. Gender differences in medication adherence among adolescent and young adult kidney transplant recipients. Transplantation [Internet] 2018 [acesso em 16 ago 2018]. Disponível em: https://www.ncbi.nlm.nih.gov/ pubmed/29994983.

14. Dias A, et al. Patients' beliefs about medicines and adherence to medication in ischemic heart disease. Atencion primaria [Internet]. 2014 [acesso em 10 ago 2018]; 46:101106. Disponivel em: https://www.researchgate.net/profile/ Antonio_Dias7/publication/269187445_Patients'_beliefs_ about_medicines_and_adherence_to_medication_in_ischemic_heart_disease/links/5721efee08ae5c4373ac2e89/ Patients-beliefs-about-medicines-and-adherence-to-medication-in-ischemic-heart-disease.pdf.

15. Arruda DCJ, et al. Fatores associados a não adesão medicamentosa entre idosos de um ambulatório filantrópico do Espírito Santo. Revista Brasileira de Geriatria e Gerontologia [Internet]. 2015 [acesso em 23 set 2018]; 18(2):327-337. Disponível em: http://www.redalyc.org/ pdf/4038/403842247010.pdf.

16. Silva LACD, Souza LEAD, Ganassoli C. Qualidade de vida na terceira idade: prevalênciade fatores intervenientes. Rev. Soc. Bras. Clín. Méd [Internet]. 2017 [acesso em 23 set 2018]; 15(3):146-149. Disponivel em: http://docs.bvsalud.org/biblioref/2017/11/875192/sbcm_153_146-149.pdf.

17. Bravin AM, Trettene ADS, Cavalcante RDS, Banin VB, Paula NADMR, Saranholi T, Andrade LGMD. Influence of spirituality on renal function of kidney transplant patients. Acta Paulista de Enfermagem [Internet]. 2017 [acesso em 23 set 2018]; 30(5):504-511. Disponível em: http://www.scielo. br/scielo.php?pid=S0103-21002017000500504\&script=sci_arttext.

18. Carvalho MFC, et al. Polifarmácia entre idosos do município de São Paulo-Estudo SABE.Revista Brasileira de Epidemiologia [Internet]. 2012 [acesso em 14 set 2018]; 15:817-827. Disponivel em: https://www.scielosp.org/scielo. php?pid=S1415-790X2012000400013\&script=sci_arttext\&tlng=es.

19. Silva ACSS, et al. Complexidade da farmacoterapia pós-transplante renal: influência na adesão ao tratamento. Revista Eletrônica de Farmácia [Internet]. 2018 jan. [acesso em 14 set 2018];14(3):1804-1808. Disponivel em: https://www.revistas. ufg.br/REF/article/view/44894.

20. Mistry N, Keepanasseril A, Wilczynski NL, Nieuwlaat R, Ravall M, Haynes RB. Patient Adherence Review Team. Technology-mediated interventions for enhancing medication adherence. Journal of the American Medical Informatics Association [Internet]. 2015 [acesso em 14 set 2018]; 22(e1):e177-e193. Disponivel em: https://academic.oup.com/jamia/article/22/e1/ e177/703258.

21. Weng LC, et al. Factors that determine self reported immunosuppressant adherence in kidney transplant recipients: a cor- relational study. Journal of advanced nursing [Internet]. 2017 [acesso em 10 ago 2018]; 79(1):228-239. Disponivel em: https://onlinelibrary.wiley.com/doi/abs/10.1111/jan.13106.

22. Jindal RM, Neff KC, Abbott FP, et al. Association between Depression and Nonadherence in Recipients of Kidney Transplants: Analysis of the United States Renal Data System. Transplant Proc [Internet]. 2009 nov. [acesso em 23 set 2018]; 41(9):3662-66. Disponivel em: https://www.ncbi.nlm.nih.gov/ pubmed/19917363.

23. Santos ASM, Oliveira NCS, Lustosa VM, Caldas DRC, Sampaio FA. Relação entre a composição corporal e o estado nutricional de pacientes sob tratamento de hemodiálise. Revista Ciência \& Saberes-Facema [Internet]. 2015 [acesso em 23 set 2018]; 1(2):119-123. Disponivel em: http://www.facema.edu. br/ojs/index.php/ReOnFacema/article/view/56/30.

24. Subtil MML, et al. 0 relacionamento interpessoal e à adesão na fisioterapia. Fisioterapia em Movimento [Internet]. 2017 [acesso em 23 set 2018]; 24(4). Disponivel em: https://periodicos.pucpr.br/index.php/fisio/article/view/21187.

25. Soares FC, Aguiar IA, Furtado NP, Carvalho RF, Torres RA, Segheto W, Costa, JA. Prevalência de hipertensão arterial e diabetes mellitus em portadores de doença renal crônica em tratamento conservador do serviço Ubaense de Nefrologia. Revista Científica FAGOC-Saúde [Internet]. 2018 [acesso em 23 set 2019]; 2(2):21-26. Disponivel em:http://revista.fagoc.br/index. php/saude/article/view/232.

26. Bittencourt ZZLDC, Alves Filho G, Mazzali M, Santos NRD. Qualidade de vida em transplantados renais: importância do enxerto funcionante. Revista de Saúde Pública [Internet]. 2014 [acesso em 27 set 2018]; 38:732-734. Disponivel em: https://www.scielosp.org/scielo.php?pi$\mathrm{d}=$ S0034-89102004000500018\&script=sci_arttext\&tlng=es. 27. Ravagnani LMB, Domingos NAM, Miyazaki MCOS. Qualidade de vida e estratégias de enfrentamento em pacientes submetidos a transplante renal. Estudos de Psicologia [Internet]. 2007 [acesso em 11 out 2018]; 12(2):177-184. Disponível em: http://www.scielo.br/pdf/epsic/v12n2/a10v12n2.

28. Brito DCS, et al. Stress, coping and adherence to immunosuppressive medications in kidney transplantation: a comparative study. São Paulo Medical Journal [Internet]. 2016 [acesso em 10 dez 2018]; 134(4):292-299. Disponivel em: http://www.scielo. br/scielo.php?pid=S1516-31802015005008106\&script=sci_arttext.

29. Marsicano EO, Fernandes NS, Colugnati FA, Fernandes NM, De Geest S, Sanders-Pinheiro H. Correlatos multiníveis da não adesão em pacientes transplantados renais que se beneficiam da cobertura total de custos para imunossupressores: um estudo transversal. PLoS One [Internet]. 2015 Nov [acesso em 10 dez 2018]; 10(11):e0138869. Disponível em: https://www.ncbi. nlm.nih.gov/pubmed/26619070?dopt=Abstract.

30. Dew MA, DiMartini AF, Vito Dabbs A, Myaskovsky L, Steel J, Unruh $M$, et al. Taxas e fatores de risco para a não adesão ao regime médico após transplante de órgão sólido em adultos. Transplantação [Internet]. 2007 [acesso em 17 set 2019]; 83(7):858-73. Disponivel em: https://www.ncbi.nlm.nih.gov/ pubmed/17460556?dopt=Abstract. 